\title{
Allelic drop-out is a common phenomenon reducing the diagnostic yield of PCR-based target sequencing
}

Shestak A.G.

Petrovsky National Research Center of Surgery, Moscow, Russia

Cardiac Electrophysiology Research

Center, Rajaie Cardiovascular Medical

and Research Center, Iran University of Medical
Bukaeva A.A.

Petrovsky National Research Center of Surgery, Moscow, Russia

Cardiac Electrophysiology Research

Center, Rajaie Cardiovascular Medical

and Research Center, Iran University of Medical

Saber S.

Petrovsky National Research Center of Surgery, Moscow, Russia

Cardiac Electrophysiology Research

Center, Rajaie Cardiovascular Medical

and Research Center, Iran University of

Medical
Zaklyazminskaya E.V. Petrovsky National Research Center of Surgery, Moscow, Russia

Cardiac Electrophysiology Research Center, Rajaie Cardiovascular Medical and Research Center, Iran University of Medical

\begin{abstract}
Allelic drop-out (ADO) is a known phenomenon of selective allele amplification representing the potential problem of correct DNA diagnostics. The results demonstrate the incidence of ADO reducing the diagnostic yield of PCRbased target sequencing
\end{abstract}

\section{Motivation and aim}

Allelic drop-out (ADO) is a known phenomenon of selective allele amplification representing the potential problem of correct DNA diagnostics. Both NGS and Sanger sequencing are PCR-based methods, Sanger sequencinq is used to verify NGS results. The aim of this study is to demonstrate the incidence of ADO reducing the diagnostic yield in primary cardiomyopathy genetic testing via semiconductor NGS and Sanger sequencing of target gene panels.

\section{Methods}

We have developed 3 AmpliSeq custom gene panels for mutational screening: " $\mathrm{K}^{+} / \mathrm{Na}^{+}$ion channels", "Desmosomal proteins", "Sarcomeric proteins", contains 1049 primer pairs (37 genes) totally, $152 \mathrm{~kb}$. About 140 probands were screened with at least one of these gene panels. AmpliSeq sequences were analyzed in silico and visually compared with Sanger control sequences, noting the facts of heterozygosity loss.

\section{Results}

We have detected 12 ADO cases both in Sanger ( 5 cases) and AmpliSeq (7 cases) sequencing data. All ADO events happened due to frequent or rare SNVs in the oligoprimer annealing sites and were detected due to mismatch in frequent SNPs zygosity nearby. Three pathogenic variants would be missed if were not revealed by re-sequencing with alternative method and alternative oligos.

\section{Conclusion}

All PCR-based methods have a risk of ADO leading to a decrease of diagnostic yield of genetic testing. ADO can theoretically affect $1 \%$ amplicons. It seems that real scope of ADO might be much higher and depends on numbers of primer pairs. The software for ADO detection is needed.

\section{ACKNOWLEDGMENT}

This work was supported by Russian Science Foundation grant No. 16-15-10421. 